\title{
WEAK COMPACTNESS AND VECTOR MEASURES
}

\author{
R. G. BARTLE, N. DUNFORD AND J. SCHWARTZ
}

Introduction. It is the purpose of this paper to develop a Lebesgue theory of integration of scalar functions with respect to a countably additive measure whose values lie in a Banach space. The class of integrable functions reduces to the ordinary space of Lebesgue integrable functions if the measure is scalar valued. Convergence theorems of the Vitali and Lebesgue type are valid in the general situation. The desirability of such a theory is indicated by recent developments in spectral theory.

In $\$ 1$ two criteria for the conditional weak compactness of subsets of the Banach space of countably additive measures on a $\sigma$-field $\Sigma$ are derived. Their force is sufficient to allow us to conclude, in $\$ 2$, that if $\mu$ is a countably additive measure on $\Sigma$ with values in a Banach space, then there exists a positive scalar measure $\nu$ on $\Sigma$ with respect to which $\mu$ is $\nu$-continuous (i.e., $\nu$-absolutely continuous). This permits the development of the integration theory.

As an example of an elementary application of the integration theory we give, in $\$ 3$, the "vector" generalization of the celebrated Riesz theorem on the representation of linear functionals on a space of continuous functions. This yields representation theorems for the general, the weakly compact, and the compact operators on a space of continuous functions. Some of these results are related to theorems of Gelfand (6) and Grothendieck (7).

Our notation and terminology are standard. We mention specifically that the weak topology of a Banach space $\mathfrak{X}$ is the topology induced by its adjoint space $\mathfrak{X}^{*}$ in the familiar fashion, even though $\mathfrak{X}$ itself may be the adjoint of some other space. By the $\mathfrak{X}$ topology of $\mathfrak{X}^{*}$ we mean the topology on $\mathfrak{X}^{*}$ which has as a typical neighbourhood of the origin, the set $\left\{x^{*} \in \mathfrak{X}^{*}|| x^{*}\left(x_{i}\right) \mid<1\right.$, $i=1, \ldots, n\}$.

1. Weakly compact sets of measures. Let $S$ be an abstract set and $\Sigma$ be a $\sigma$-field (i.e., $\sigma$-algebra) of subsets of $S$; sets in $\Sigma$ will frequently be called the measurable subsets of $S$.

1.1. Definition. By ca $(\Sigma)$ we denote the set of all countably additive real or complex valued measures defined on $\Sigma$ having finite variation. A generic element of $\mathrm{ca}(\Sigma)$ will ordinarily be denoted by the letter $\lambda$; positive measures by $\nu$. The symbol $|\lambda|(E)$ denotes the total variation of $\lambda$ over the set $E \in \Sigma$. The symbol $|\lambda|$ represents the total variation of $\lambda$ over the entire space $S$.

Received June 20, 1954; in revised form February 20, 1955. The research contained in this paper was done under contract NONR609(04) with the Office of Naval Research. 
With $|\lambda|$ as the norm, ca $(\Sigma)$ forms a real or complex Banach space. It is the purpose of this section to characterize the subsets of $\mathrm{ca}(\Sigma)$ which are conditionally weakly compact. We shall make free use of the theorem of Eberlein (5) asserting that such sets $K$ are precisely those which are weakly sequentially compact in the sense that an arbitrary sequence from $K$ has a subsequence which converges weakly to an element of ca $(\Sigma)$. We observe that if a sequence $\left\{\lambda_{n}\right\}$ converges weakly, then it is bounded and $\left\{\lambda_{n}(E)\right\}$ is a convergent set of scalars for each $E \in \Sigma$.

Finally, we mention that we will make essential use of the Vitali-Hahn-Saks theorem $\left(15\right.$, p. 967): Let $\nu$ be a finite positive measure on $\Sigma$, let $\left\{\lambda_{n}\right\} \subset \mathrm{ca}(\Sigma)$ be such that $\lim \lambda_{n}(E)$ exists for each $E \in \Sigma$, and let

$$
\lim _{\nu(E) \rightarrow 0} \lambda_{n}(E)=0
$$

for each $n$. It follows that this last limit exists uniformly in $n$, and if $\lambda$ is the set function defined by

for $E \in \Sigma$, then $\lambda \in \mathrm{ca}(\Sigma)$.

$$
\lambda(E)=\lim _{n \rightarrow \infty} \lambda_{n}(E)
$$

1.2. Lemma. If $\left\{\lambda_{i}\right\}$ is a sequence in $\mathrm{ca}(\Sigma)$, there is a positive measure $\nu \in \mathrm{ca}(\Sigma)$ such that

$$
\lim _{\nu(E) \rightarrow 0} \lambda_{i}(E)=0, \quad i=1,2, \ldots
$$

Proof. The measure defined by

$$
\nu(E)=\sum_{i=1}^{\infty} 2^{-i} \frac{\left|\lambda_{i}\right|(E)}{1+\left|\lambda_{i}\right|}, \quad E \in \Sigma,
$$

has the property that if $\nu(E)=0$ then $\left|\lambda_{i}\right|(E)=0$. The conclusion then follows (8, p. 125).

1.3. Theorem. ${ }^{1}$ For a set $K \subset \mathrm{Ca}(\Sigma)$ to be conditionally weakly compact it is necessary and sufficient that

(1) the set $K$ is bounded, and

(2) if $\left\{E_{i}\right\}$ is a sequence in $\Sigma$ which decreases to the void set, then

uniformly for $\lambda \in K$.

$$
\lim _{i \rightarrow \infty} \lambda\left(E_{i}\right)=0 \text {, }
$$

Proof. The necessity of (1) is a consequence of the uniform boundedness theorem. To show that (2) is necessary we proceed indirectly, and suppose that there exists a positive number $\epsilon$, a sequence $\left\{E_{i}\right\} \subset \Sigma$ with $E_{i} \downarrow \phi$, and a sequence $\left\{\lambda_{i}\right\} \subseteq K$ such that

$$
\left|\lambda_{i}\left(E_{i}\right)\right|>\epsilon, \quad i=1,2, \ldots
$$

${ }^{1}$ The reader should observe the similarity between this theorem and some of the criteria, established by Grothendieck (7, p. 146), for weak compactness in the space of bounded regular Radon) measures. 
Since $K$ is assumed to be weakly compact, we may suppose that $\left\{\lambda_{i}\right\}$ is weakly convergent so that $\lim \lambda_{i}(E)$ exists for all $E \in \Sigma$. Construct $\nu \in \operatorname{ca}(\Sigma)$ as in the lemma; by the Vitali-Hahn-Saks theorem

$$
\lim _{\nu(E) \rightarrow 0} \lambda_{i}(E)=0, \quad \text { uniformly for } i=1,2, \ldots
$$

Since $E_{i} \downarrow \phi$, it follows that $\nu\left(E_{i}\right) \rightarrow 0$ and hence $\lambda_{i}\left(E_{i}\right) \rightarrow 0$, which contradicts the supposition and establishes the necessity of (2).

To prove the sufficiency, let $\left\{\lambda_{m}\right\}$ be an arbitrary sequence in $K$. Construct a positive measure $\nu \in \mathrm{ca}(\Sigma)$ as in the lemma. Let ca $(\Sigma ; \nu)$ be the closed linear manifold in ca $(\Sigma)$ consisting of measures $\lambda$ which are $\nu$-continuous, i.e. such that

$$
\lim _{\nu(E) \rightarrow 0} \lambda(E)=0 .
$$

By the Radon-Nikodmý theorem (8, p. 128) there is an isometric isomorphism between the space ca $(\Sigma, \nu)$ and the space $L(S, \Sigma, \nu)$ of $\nu$-integrable functions, the correspondence $\lambda \leftrightarrow f$ being given by the formula

$$
\lambda(E)=\int_{E} f(s) \nu(d s), \quad E \in \Sigma .
$$

Denote the functions corresponding to the $\left\{\lambda_{m}\right\}$ by $\left\{f_{m}\right\}$. Let $\left\{G_{n} \mid n=1,2, \ldots\right\}$ be a basis for the open sets in the scalar field and let $E_{m n}=f_{m}{ }^{-1}\left(G_{n}\right)$.

Let $\Sigma_{1}$ be the $\sigma$-field generated by the sets $\left\{E_{m n}\right\}$. Observe that each $f_{m}$ is measurable with respect to $\Sigma_{1}$ and thus is in the space $L\left(S, \Sigma_{1}, \nu\right)$. Let $\Sigma_{2}$ be the field generated by the sets $\left\{E_{m n}\right\}$; it is known $\left(8\right.$, p. 23) that $\Sigma_{2}$ consists of a countable number of sets. By a diagonal process, pick a subsequence

such that

$$
\left\{\lambda_{m_{k}}\right\}
$$

$$
\lim _{k} \lambda_{m_{k}}(E)
$$

exists for all $E \in \Sigma_{2}$. Now let $\Sigma_{3}$ be the class of all subsets in $\Sigma_{1}$ for which

$$
\lim _{k} \lambda_{m_{k}}(E)
$$

exists. Evidently, $\Sigma_{2} \subseteq \Sigma_{3} \subseteq \Sigma_{1}$.

We wish to show that $\Sigma_{3}=\Sigma_{1}$ and will accomplish this by demonstrating that $\Sigma_{3}$ is a monotone class (8, p. 27). Let $\left\{E_{i}\right\} \subset \Sigma_{3}$ and suppose that $E_{i} \uparrow E$. Then $E-E_{i} \downarrow \phi$, so by condition (2) we have that

$$
\lim _{i} \lambda_{m_{k}}\left(E_{i}\right)=\lambda_{m_{k}}(E)
$$

uniformly with respect to $k=1,2, \ldots$ But since $E_{i} \in \Sigma_{3}$,

$$
\lim _{k} \lambda_{m_{k}}\left(E_{i}\right)
$$

exists for $i=1,2, \ldots$ It follows that

$$
\lim _{k} \lambda_{m_{k}}(E)
$$

exists and hence $E \in \Sigma_{3}$. Thus $\Sigma_{3}$ is a monotone class and $\Sigma_{3}=\Sigma_{1}$. 
We now prove that

$$
\left\{\lambda_{m_{k}}\right\}
$$

is a weakly convergent sequence in $\mathrm{ca}(\Sigma)$. By the Hahn-Banach theorem $(8 a$, p. 19$)$ it is enough to prove that this sequence is weakly convergent in the space $\operatorname{ca}(\Sigma ; \nu)$, and in view of the isometric isomorphism between this space and $L(S, \Sigma, \nu)$ it suffices to show that

$$
\left\{f_{m_{k}}\right\}
$$

is a weakly convergent sequence in $L(S, \Sigma, \nu)$ or even in the subspace $L\left(S, \Sigma_{1}, \nu\right)$. Since this sequence is bounded it is enough to prove convergence on a fundamental set of continuous linear functionals on $L\left(S, \Sigma_{1}, \nu\right)$, such as the characteristic functions of subsets of $\Sigma_{1}$. But the convergence on this fundamental set is equivalent to the convergence of

$$
\left\{\lambda_{m_{k}}\right\}
$$

on $\Sigma_{1}$, which has already been established. This proves the sufficiency of the conditions.

We shall also need the following criterion:

1.4. Theorem. For a set $K \subset \mathrm{Ca}(\Sigma)$ to be conditionally weakly compact it is necessary and sufficient that

(1) the set $K$ is bounded, and

$\left(2^{\prime}\right)$ there exists a positive $\nu \in \mathrm{ca}(\Sigma)$ such that

$$
\lim _{\nu(E) \rightarrow 0} \lambda(E)=0 \text {, }
$$

uniformly for $\lambda \in K$.

Proof. To prove the necessity of $\left(2^{\prime}\right)$, we shall show that for any positive $\epsilon$, there is a positive $\delta(\epsilon)$ and a finite set $\left\{\lambda_{1}, \ldots, \lambda_{p}\right\} \subseteq K$ such that if $\left|\lambda_{i}\right|(E)<\delta(\epsilon)$ for $i=1, \ldots, p$ then $|\lambda(E)|<\epsilon$ for $\lambda \in K$. Suppose that this statement is false for some $\epsilon$. Let $\lambda_{1} \in K$ be arbitrary; then there exists a set $E_{1} \in \Sigma$ and a $\lambda_{2} \in K$ such that

$$
\left|\lambda_{1}\right|\left(E_{1}\right)<2^{-1}, \quad\left|\lambda_{2}\left(E_{1}\right)\right| \geqslant \epsilon .
$$

By induction, construct sequences $\left\{\lambda_{i}\right\} \subseteq K$, and $\left\{E_{i}\right\} \subseteq \Sigma$ such that

$$
\begin{array}{lr}
\left|\lambda_{i}\right|\left(E_{j}\right)<2^{-j}, & 1 \leqslant i \leqslant j<\infty, \\
\left|\lambda_{j+1}\left(E_{j}\right)\right| \geqslant \epsilon, & 1 \leqslant j<\infty .
\end{array}
$$

We shall suppose that the sequence $\left\{\lambda_{i}\right\}$ converges weakly in ca $(\Sigma)$. Construct a positive measure $\nu^{\prime} \in \mathrm{ca}(\Sigma)$ by Lemma 1.2. By the Vitali-Hahn-Saks theorem

$$
\lim _{\nu^{\prime}(E) 0} \lambda_{i}(E)=0, \quad \text { uniformly for } i=1,2, \ldots
$$

From the construction of $\nu^{\prime}$ and $(*)$ we have that $\nu^{\prime}\left(E_{j}\right) \leqslant 2.2^{-j}$. On the other hand, $\left|\lambda_{j+1}\left(E_{j}\right)\right| \geqslant \epsilon$, which is a contradiction and establishes the validity of the statement. Now let $\epsilon$ take on the values $2^{-n}, n=1,2, \ldots$, and let $\delta\left(2^{-n}\right)$ 
be the corresponding numbers and $\left\{\lambda_{n p} \mid 1 \leqslant p \leqslant P_{n}, 1 \leqslant n<\infty\right\} \subseteq K$ the measures whose existence has been assured. Let $\nu$ be the positive measure in ca $(\Sigma)$ defined by

$$
\nu(E)=\sum_{n=1}^{\infty} 2^{-n} \sum_{p=1}^{P_{n}}-\frac{1}{P_{n}} \frac{\left|\lambda_{n p}\right|(E)}{1+\left|\lambda_{n p}\right|}, \quad E \in \Sigma .
$$

Then if $\nu(E)<\delta\left(2^{-n}\right)\left\{2^{n} P_{n}(1+M)\right\}^{-1}$, where $M=\sup _{\lambda \in K}|\lambda|$, it is evident that $\left|\lambda_{n p}\right|(E)<\delta\left(2^{-n}\right)\left(p=1, \ldots, P_{n}\right)$ and hence $|\lambda(E)|<2^{-n}$ for all $\lambda \in K$.

To prove the sufficiency of the conditions, we observe that $\left(2^{\prime}\right)$ implies condition (2) of the preceding theorem, so the proof of the theorem is complete. The preceding proof establishes the partial converse:

1.5. Corollary. If $K$ is a conditionally weakly compact subset of $\mathrm{ca}(\Sigma)$, then there exists a positive $\nu \in \mathrm{ca}(\Sigma)$ satisfying $\left(2^{\prime}\right)$ and such that $\nu(E)<\epsilon$ whenever $E \in \Sigma$ is such that $|\lambda|(E)<\epsilon$ for all $\lambda \in K$.

2. Integration with respect to a vector measure. Let $\mathfrak{X}$ be a real or complex Banach space, and $\mathfrak{X}^{*}$ its conjugate space. We shall be concerned with a fixed additive set function $\mu$ defined on a $\sigma$-field $\Sigma$ of subsets of a set $S$ and taking values in $\mathfrak{X}$.

2.1. Definition. The additive set function $\mu: \Sigma \rightarrow \mathfrak{X}$ is called a vector measure if $x^{*} \mu \in \operatorname{ca}(\Sigma)$ for every $x^{*} \in \mathfrak{X}^{*}$; thus the set functions $\left\{x^{*} \mu \mid x^{*} \in \mathfrak{X}^{*}\right\}$ on $\Sigma$ to the scalar field are finite valued and countably additive.

If $\mu$ is a vector measure, we define a non-negative set function $\|\mu\|$ on $\Sigma$, called the semi-variation of $\mu$, by

$$
\|\mu\|(E)=\sup \left|\sum_{i=1}^{n} \alpha_{i} \mu\left(E_{i}\right)\right|, \quad E \in \Sigma,
$$

where the supremum is taken over all finite collections of scalars with $\left|\alpha_{i}\right| \leqslant 1$ and all partitions of $E$ into a finite number of disjoint measurable sets. Evidently $\|\mu\|(E) \leqslant\|\mu\|(F)$ if $E \subseteq F$; further,

$$
\|\mu\|\left(\bigcup_{i=1}^{\infty} E_{i}\right) \leqslant \sum_{i=1}^{\infty}\|\mu\|\left(E_{i}\right)
$$

for every sequence of measurable sets $\left\{E_{i}\right\}$, and the inequality may be strict.

2.2. Lemma. If $\mu: \Sigma \rightarrow \mathfrak{X}$ is a vector measure and if $\left\{E_{i}\right\}$ is a sequence of disjoint measurable sets, then

$$
\mu\left(\bigcup_{i=1}^{\infty} E_{i}\right)=\sum_{i=1}^{\infty} \mu\left(E_{i}\right),
$$

where the series converges unconditionally in the norm of $\mathfrak{X}$. The semi-variation of $\mu$ on $S$ is finite; in fact, if $E \in \Sigma$, then

$$
\|\mu\|(E) \leqslant 4 \sup \{|\mu(F)| \mid F \in \Sigma, F \subseteq E\}<\infty .
$$


Proof. The first fact is due to Pettis $(11 ; 12)$. Given $x^{*} \in \mathfrak{X}^{*}, x^{*} \mu$ is a finite valued measure, so $\sup \left\{\left|x^{*} \mu(F)\right| \mid F \in \Sigma, F \subseteq E\right\}$ is finite for each $x^{*} \in \mathfrak{X}^{*}$. By the Uniform Boundedness theorem (8a, p. 26),

$$
\sup \{|\mu(F)| \mid F \in \Sigma, F \subseteq E\}<\infty .
$$

On the other hand,

$$
\|\mu\|(E)=\sup _{\left|x^{*}\right| \leqslant 1}\left|x^{*} \mu\right|(E) \leqslant 4 \sup _{\left|x^{*}\right| \leqslant 1} \sup _{F \subseteq E}\left|x^{*} \mu(F)\right|,
$$

so the conclusion follows from the Hahn-Banach theorem (8a, p. 19).

The next lemma and its corollary are crucial for our purposes.

2.3. Lemma. The set of numerical measures $\left\{x^{*} \mu\left|x^{*} \in \mathfrak{X}^{*},\right| x^{*} \mid \leqslant 1\right\}$ is conditionally weakly compact as a subset of $\mathrm{ca}(\Sigma)$.

Proof. Let $\left\{E_{i}\right\}$ be a decreasing sequence of sets in $\Sigma$ with void intersection. Since $\mu$ is countably additive in the strong topology, $\lim \mu\left(E_{i}\right)=0$ in this topology. Consequently, $\lim x^{*} \mu\left(E_{i}\right)=0$ uniformly for $\left|x^{*}\right| \leqslant 1$. By Theorem 1.3 the desired result follows.

2.4. Corollary. If $\mu$ is a vector measure, then there is a positive measure $\nu \in \mathrm{ca}(\Sigma)$ such that

$$
\lim _{\nu(E) \rightarrow 0} \mu(E)=0, \quad \lim _{\nu(E) \rightarrow 0}\|\mu\|(E)=0 .
$$

The measure $\nu$ may be chosen such that $\nu(E)<\epsilon$ whenever $\|\mu\|(E)<\epsilon$.

Proof. The first equation follows from Lemma 2.3, Theorem 1.4, and the Hahn-Banach theorem; the second follows from the first and Lemma 2.2. The final statement is a consequence of Corollary 1.5.

We now proceed to develop a theory of integration of scalar functions with respect to the vector measure $\mu$. A $\mu$-null set is a set $E \in \Sigma$ for which $\|\mu\|(E)=0$; the term $\mu$-almost everywhere refers to the complement of a $\mu$-null set. From the countable sub-additivity of $\|\mu\|$ it follows that the union of a countable number of $\mu$-null sets is also a $\mu$-null set. A scalar function $f$ defined on $S$ is measurable if for every Borel set $B$ of scalars, $f^{-1}(B)$ is an element of $\Sigma$. A function is simple if it is a finite linear combination of characteristic functions of measurable sets.

If $f$ is the simple function

$$
\sum_{i=1}^{n} \alpha_{i} \chi_{E_{i}}, \quad E_{i} \in \Sigma,
$$

then we define the integral of $f$ over a set $E \in \Sigma$ by the equation

$$
\int_{E} f(s) \mu(d s)=\sum_{i=1}^{n} \alpha_{i} \mu\left(E \cap E_{i}\right)
$$


The integral of a simple function is independent of the representation of the function as a linear combination of characteristic functions. Obviously, integration of simple functions over $E$ is a linear operation. Also, the integral of a simple function is a countably additive set function with values in $\mathfrak{X}$. If $f$ is a simple function and if $E$ is in $\Sigma$ then evidently

$$
\left|\int_{E} f(s) \mu(d s)\right| \leqslant \sup _{s \in E}|f(s)| \cdot\|\mu\|(E) .
$$

If $f$ is an arbitrary measurable function, we define the $\mu$-essential supremum of $f$ on $E$ to be the infimum of those numbers $A$ for which $\{s \in E|| f(s) \mid>A\}$ is a $\mu$-null set. If

$$
\mu \text {-ess } \sup _{s \in E}|f(s)|<\infty,
$$

then we say that $f$ is $\mu$-essentially bounded on the set $E \in \Sigma$.

2.5. Definition. A measurable function $f$ is said to be $\mu$-integrable if there exists a sequence $\left\{f_{n}\right\}$ of simple functions such that

(1) $f_{n}(s) \rightarrow f(s) \mu$-almost everywhere, and

(2) the sequence

$$
\left\{\int_{E} f_{n}(s) \mu(d s)\right\}
$$

converges in the norm of $\mathfrak{X}$ for each $E \in \Sigma$.

The limit of this sequence of integrals is defined to be the integral of $f$ with respect to $\mu$ over the set $E \in \Sigma$, in symbols

$$
\int_{E} f(s) \mu(d s) \text {. }
$$

2.6. THEOREM. (a) If $E \in \Sigma$ and $f$ is $\mu$-integrable, the integral of $f$ with respect to $\mu$ over $E$ is an unambiguously defined element of $\mathfrak{X}$;

(b) the integrable functions form a linear space and for $E$ in $\Sigma$ the integral $\int_{E} f(s) \mu(d s)$ is a linear map of this space into $\mathfrak{X}$ :

(c) if $f$ is a measurable function which is $\mu$-essentially bounded on $E$, then $f$ is $\mu$-integrable and

$$
\left|\int_{E} f(s) \mu(d s)\right| \leqslant\{\mu \text {-ess sup }|f(s)|\}\{\|\mu\|(E)\} ;
$$

(d) if $f$ is $\mu$-integrable, then $\int_{E} f(s) \mu(d s)$ is a countable additive function on $\Sigma$ to $\mathfrak{X}$;

(e) if $f$ is $\mu$-integrable, then

$$
\lim _{\|\mu\|(E) \rightarrow 0} \int_{E} f(s) \mu(d s)=0 ;
$$

(f) if $U$ is a bounded linear operator from $\mathfrak{X}$ into a second Banach space $\mathfrak{Y}$, then $U_{\mu}$ is a vector measure from $\Sigma$ to $\mathfrak{Y}$ and for any $\mu$-integrable function $f$ and $E \in \Sigma$, we have

$$
U\left\{\int_{E} f(s) \mu(d s)\right\}=\int_{E} f(s) U \mu(d s) .
$$


Proof. To prove (a), let $\left\{f_{n}\right\}$ and $\left\{g_{n}\right\}$ be two sequences of simple functions as in Definition 2.5; we are required to show that the two sequences of integrals approach the same limit. We define $h_{n}(s)=0$, if $s$ is a point at which either $\left\{f_{n}(s)\right\}$ or $\left\{g_{n}(s)\right\}$ fails to converge to $f(s)$, and set $h_{n}(s)=f_{n}(s)-g_{n}(s)$ otherwise. It is evident that $\left\{h_{n}\right\}$ converges to zero everywhere, and that $\left\{\int_{E} h_{n}(s) \mu(d s)\right\}$ converges in the norm of $\mathfrak{X}$ for $E \in \Sigma$. We must show that this sequence of integrals converges to the zero element of $\mathfrak{X}$.

Let $\nu$ be a positive measure described in Corollary 2.4. Clearly, since each $h_{n}$ is a simple function,

$$
\lim _{\nu(E) \rightarrow 0} \int_{E} h_{n}(s) \mu(d s)=0, \quad n=1,2, \ldots ;
$$

further, the sequence of integrals $\left\{\int_{E} h_{n}(s) \mu(d s)\right\}$ converges for each $E \in \Sigma$, so by the Vitali-Hahn-Saks theorem, ${ }^{2}$ the limit in $\left({ }^{*}\right)$ is uniform in $n$. Consequently, for each $\epsilon>0$ there exists a $\delta=\delta(\epsilon)>0$ such that if $A \in \Sigma$ and $\nu(A)<\delta$ then

$$
\left|\int_{A} h_{n}(s) \mu(d s)\right|<\epsilon, \quad n=1,2, \ldots
$$

By Egoroff's theorem (8, p. 88) there exists a set $A \in \Sigma$ with $\nu(A)<\delta$ such that $\left\{h_{n}(s)\right\}$ converges to zero uniformly for $s \in S-A$. Having specified $\epsilon$ and chosen $\delta=\delta(\epsilon)$ as above, there exists an $N=N(\epsilon)$ such that if $n \geqslant N$, then $\left|h_{n}(s)\right|<\epsilon$ for $s \in S-A$. Hence if $n \geqslant N$,

$$
\begin{aligned}
&\left|\int_{E} h_{n}(s) \mu(d s)\right| \leqslant\left|\int_{E-A^{h_{n}(s) \mu(d s)}|+|} \leqslant \epsilon\right| \mid \mu \|(S)+\epsilon . \\
& E \cap A^{h_{n}(s) \mu(d s)} \mid
\end{aligned}
$$

uniformly for $E \in \Sigma$. Thus the integral is well defined.

Statements (b) and (c) follow readily while (d) and (e) follow from their validity for simple functions, the Vitali-Hahn-Saks theorem, and Corollary 2.4. The first assertion in (f) follows from Definition 2.1. and the observation that $y^{*} U_{\mu}=\left(U^{*} y^{*}\right) \mu, y^{*} \in \mathfrak{Y}^{*}$. The second assertion follows from its validity for simple functions.

2.7. THEOREM. Let $\left\{f_{n}\right\}$ be a sequence of $\mu$-integrable functions which converges $\mu$-almost everywhere to $f$. Then $f$ is $\mu$-integrable if

$$
\lim _{\|\mu\|(E) \rightarrow 0} \int_{E} f_{n}(s) \mu(d s)=0
$$

uniformly for $n=1,2, \ldots$ In this case we have

$$
\int_{\boldsymbol{E}} f(s) \mu(d s)=\lim _{n \rightarrow \infty} \int_{\boldsymbol{E}} f_{n}(s) \mu(d s) .
$$

${ }^{2}$ That this theorem remains valid for vector measures may be seen by examining the proof in Saks (15), and has been proved directly by Alexiewicz (1, p. 19) from more general considerations. 
Proof. Let $k$ be a fixed positive integer and let $\delta_{k}>0$ be such that if $E \in \Sigma$ and $\|\mu\|(E)<\delta_{k}$, then

$$
\left|\int_{E} f_{n}(s) \mu(d s)\right|<2^{-k}, \quad n=1,2, \ldots .
$$

This implies that if $\|\mu\|(E)<\delta_{k}$, then

$$
\int_{E}\left|f_{n}(s)\right|\left|x^{*} \mu\right|(d s)<16 \cdot 2^{-k}, \quad\left|x^{*}\right| \leqslant 1, \quad n=1,2, \ldots
$$

Evidently, we may assume that $\delta_{k} \leqslant 2^{-k}$. Let $\eta_{k}>0$ be such that if $A \in \Sigma$ and $\nu(A)<\eta_{k}$ then $\|\mu\|(A)<\delta_{k}$. By Corollary 2.4, the sequence $\left\{f_{n}\right\}$ converges $\nu$-almost everywhere, and by an application of Egoroff's theorem, we can select a set $A \in \Sigma$ such that $\nu(A)<\eta_{k}$ and such that the convergence of $\left\{f_{n}\right\}$ is uniform on $S-A$. Thus if $E \in \Sigma$ and $n, m \geqslant N_{k}$, we have

$$
\begin{aligned}
\left|\int_{E}\left\{f_{n}(s)-f_{m}(s)\right\} \mu(d s)\right| & \leqslant\left|\int_{E-A}\left\{f_{n}(s)-f_{m}(s)\right\} \mu(d s)\right| \\
& +\left|\int_{E \cap A} f_{n}(s) \mu(d s)\right| \\
& +\left|\int_{E \cap A} f_{m}(s) \mu(d s)\right|<2^{-k}\{\|\mu\|(S)+2\} .
\end{aligned}
$$

But since $k$ is an arbitrary positive integer, this proves that the sequence $\left\{\int_{E} f_{n}(s) \mu(d s)\right\}$ converges in the norm of $\mathfrak{X}$ for any $E \in \Sigma$.

We now prove that $f$ is $\mu$-integrable. Let $\delta_{k}$ and $\eta_{k}$ have the same meaning as in the previous paragraph. Since each $f_{k}$ is $\mu$-integrable it follows from Egoroff's theorem that there is a simple function $g_{k}$ and a set $A_{k} \in \Sigma$ with $\nu\left(A_{k}\right)<\eta_{k}$ such that and

Let

$$
\begin{array}{rr}
\left|f_{k}(s)-g_{k}(s)\right|<2^{-k}, & s \in S-A_{k}, \\
\left|g_{k}(s)\right|<2\left|f_{k}(s)\right|, & s \in S .
\end{array}
$$

$$
B_{k}=\bigcup_{i=k}^{\infty} A_{i}
$$

so that $B_{k} \in \Sigma$ and $\left\{B_{k}\right\}$ decreases to the set

Since

$$
B=\bigcap_{k=1}^{\infty} B_{k} \text {. }
$$

$$
\|\mu\|\left(B_{k}\right) \leqslant \sum_{i=k}^{\infty}\|\mu\|\left(A_{i}\right)<\sum_{i=k}^{\infty} \delta_{i} \leqslant \sum_{i=k}^{\infty} 2^{-i}=2^{-(k-1)},
$$

it follows that $\|\mu\|(B)=0$. Now

$$
\left|f(s)-g_{k}(s)\right| \leqslant\left|f(s)-f_{k}(s)\right|+f_{k}(s)-g_{k}(s) \mid .
$$

If $s \in S-B$, then $s \in S-B_{k}$ for $k \geqslant K(s)$ and so (4) holds provided that $k \geqslant K(s)$. Since $\left\{f_{k}\right\}$ was assumed to converge $\mu$-almost everywhere to $f$, we 
conclude that the sequence $\left\{g_{k}\right\}$ converges $\mu$-almost everywhere to $f$. It remains to show that the integrals $\left\{\int_{E} g_{n}(s) \mu(d s)\right\}$ converge for $E \in \Sigma$. But

$$
\begin{aligned}
\left|\int_{E}\left\{f_{k}(s)-g_{k}(s)\right\} \mu(d s)\right| & \leqslant\left|\int_{E-A_{k}}\left\{f_{k}(s)-g_{k}(s)\right\} \mu(d s)\right| \\
& +\left|\int_{E \cap A_{k}} f_{k}(s) \mu(d s)\right|+\left|\int_{E \cap A_{k}} g_{k}(s) \mu(d s)\right| .
\end{aligned}
$$

The integral over $E-A_{k}$ is at most $2^{-k}\|\mu\|(S)$ by (4). Since $\|\mu\|\left(E \cap A_{k}\right)<\delta_{k}$, we have seen in (1) that the second term is at most $2^{-k}$. In addition, from (5) and (2),

$$
\begin{aligned}
\left|\int_{E \cap A_{k}} g_{k}(s) \mu(d s)\right| & =\sup _{\left|x^{*}\right| \leqslant 1} \mid \int_{E \cap A_{k}}{ }^{g_{k}(s) x^{*} \mu(d s) \mid} \\
& \leqslant \sup _{\left|x^{*}\right| \leqslant 1} \int_{E \cap A_{k}}\left|g_{k}(s)\right|\left|x^{*} \mu\right|(d s) \\
& \leqslant 2 \sup _{\left|x^{*}\right| \leqslant 1} \int_{E \cap A_{k}}\left|f_{k}(s)\right|\left|x^{*} \mu\right|(d s) \leqslant 32 \cdot 2^{-k} .
\end{aligned}
$$

Combining these, we conclude that

$$
\left|\int_{E}\left\{f_{k}(s)-g_{k}(s)\right\} \mu(d s)\right| \leqslant 2^{-k}\{\|\mu\|(S)+33\} .
$$

Now if $k$ is given, then combining (3) and (6) we see that if $n, m \geqslant \max \left(k, N_{k}\right)$ and $E \in \Sigma$, we have

$$
\begin{aligned}
\mid \int_{E}\left\{g_{n}(s)-\right. & \left.g_{m}(s)\right\} \mu(d s) \mid \\
& \leqslant 2 \cdot 2^{-k}\{\|\mu\|(S)+33\}+2^{-k}\{\|\mu\|(S)+2\} \\
& <M \cdot 2^{-k} .
\end{aligned}
$$

Hence the sequence of integrals of the simple functions $g_{n}$ converges for $E \in \Sigma$, so that $f$ is $\mu$-integrable.

To prove the last statement, let $h_{n}(s)=f(s)-f_{n}(s), n=1,2, \ldots$; then each $h_{n}$ is $\mu$-integrable and, by Theorem $2.6(\mathrm{e})$, equation $\left(^{*}\right)$ in the proof of that theorem is valid. The argument there applies in this case to prove that

$$
\lim _{n \rightarrow \infty}\left|\int_{E}\left\{f(s)-f_{n}(s)\right\} \mu(d s)\right|=0,
$$

which is the desired conclusion.

We now show that the theorem on dominated convergence is valid for vector measures.

2.8. THEOREM. If $\left\{f_{n}\right\}$ is a sequence of $\mu$-integrable functions which converges $\mu$-almost everywhere to $f$ and if $g$ is a $\mu$-integrable function such that $\left|f_{n}(s)\right| \leqslant g(s)$ $\mu$-almost everywhere, $n=1,2, \ldots$, then $f$ is $\mu$-integrable and

$$
\int_{E} f(s) \mu(d s)=\lim _{n \rightarrow \infty} \int_{E} f_{n}(s) \mu(d s), \quad E \in \Sigma .
$$


Proof. By the preceding theorem, we have only to show that

$$
\lim _{\|\mu\|(E) \rightarrow 0} \int_{E} f_{n}(s) \mu(d s)=0
$$

uniformly for $n=1,2, \ldots$ Now by Theorem $2.6(\mathrm{e})$, given $\epsilon>0$, choose $\delta>0$ such that if $E \in \Sigma,\|\mu\|(E)<\delta$ then

Hence if $\|\mu\|(E)<\delta$ we have

$$
\left|\int_{E} g(s) \mu(d s)\right|<\epsilon .
$$

$$
\int_{E} g(s)\left|x^{*} \mu\right|(d s)<4 \epsilon, \quad\left|x^{*}\right| \leqslant 1 .
$$

Consequently, if $n=1,2, \ldots$, and $\|\mu\|(E)<\delta$, then

$$
\begin{aligned}
\left|\int_{E^{\prime}} f_{n}(s) \mu(d s)\right| & \leqslant \sup _{\left|x^{*}\right| \leqslant 1} \int_{E^{\prime}}\left|f_{n}(s)\right|\left|x^{*} \mu\right|(d s) \\
& \leqslant \sup _{\left|x^{*}\right| \leqslant 1} \int_{E^{2}} g(s)\left|x^{*} \mu\right|(d s) \leqslant 4 \epsilon,
\end{aligned}
$$

from which the conclusion follows.

While the following theorem will not be used in the sequel, it has some independent interest.

2.9. TheOREM. The range of a vector measure $\mu: \Sigma \rightarrow \mathfrak{X}$ is a conditionally weakly compact subset of $\mathfrak{X}$.

Proof. We shall prove that if $\Re=\{\mu(E) \mid E \in \Sigma\}$ is regarded as a subset of $\mathfrak{X}^{* *}$ in the natural embedding, then $\Re$ is conditionally compact in the weak topology of $\mathfrak{X}^{* *}$. Since the embedding of $\mathfrak{X}$ in $\mathfrak{X}^{* *}$ is closed in this topology, the statement will follow. Now we have seen in Lemma 2.3 that the mapping $U: \mathfrak{X}^{*} \rightarrow \mathrm{ca}(\Sigma)$ defined by $U x^{*}=x^{*} \mu$ is a weakly compact operator; hence the adjoint operator $U^{*}: \mathrm{ca}^{*}(\Sigma) \rightarrow \mathfrak{X}^{* *}$ is also weakly compact. But the unit sphere of $\mathrm{ca}^{*}(\Sigma)$ certainly contains the linear functionals $\left\{\phi_{E} \mid E \in \Sigma\right\}$ defined by $\phi_{E}(\lambda)=\lambda(E), \lambda \in \mathrm{ca}(\Sigma)$. Clearly $U^{*}\left\{\phi_{E} \mid E \in \Sigma\right\}=\{\mu(E) \mid E \in \Sigma\}$, this latter set being regarded in $\mathfrak{X}^{* *}$, and $\Re$ is therefore conditionally weakly compact.

3. Representation of operators on continuous functions. Throughout this section $S$ will denote a compact Hausdorff space and $\Sigma$ its Borel field, i.e., the $\sigma$-field generated by the closed sets of $S$. The $\sigma$-field generated by the closed $G$ sets of $S_{\delta}$ is called the Baire field of $S$ and is denoted by $\Sigma_{0}$. The Banach space of all continuous scalar valued functions on $S$ with the supremum norm is written $C(S)$. The Riesz representation theorem (see Kakutani (10)) asserts that the conjugate space of $C(S)$ is isometrically isomorphic to either ca $\left(\Sigma_{0}\right)$ or to the space $R(S)$ of regular Borel measures on $S$, where the norm in these measure spaces is the total variation over $S$. 
Let $T$ be a bounded linear operator mapping $C(S)$ into a Banach space $\mathfrak{X}$. We say that $T$ is compact, or that it is weakly compact, if $T$ maps bounded sets of $C(S)$ into subsets of $\mathfrak{X}$ which are conditionally compact, or which are conditionally weakly compact, respectively. We shall make frequent use of the fact that $T$ is compact (or weakly compact) if and only if its adjoint $T^{*}$ is compact (or weakly compact). In addition, $T$ is weakly compact if and only if its second adjoint $T^{* *}$ maps $C^{* *}(S)$ into $\mathfrak{X}$ (more precisely, if the range of $T^{* *}$ is contained in the natural embedding of $\mathfrak{X}$ in $\left.\mathfrak{X}^{* *}\right)$. For the proofs of the above facts, see $(2 ; \mathbf{4})$.

Motivated by the Riesz theorem we are led to inquire when an operator $T: C(S) \rightarrow \mathfrak{X}$ may be represented by an integral with respect to a vector measure-we shall see that this is possible if and only if $T$ is weakly compact. First, however, it will be convenient to give a representation of the general operator.

3.1. Theorem. If $T$ is an operator on $C(S)$ to $\mathfrak{X}$, there exists a unique set function $\mu: \Sigma \rightarrow \mathfrak{X}^{* *}$ such that

(a) $\mu(\cdot) x^{*} \in R(S)$ for each $x^{*} \in \mathfrak{X}^{*}$;

(b) the mapping $x^{*} \rightarrow \mu(\cdot) x^{*}$ of $\mathfrak{X}^{*}$ into $R(S)$ is continuous with the $\mathfrak{X}$ and $C(S)$ topologies in these spaces, respectively;

(c) $x^{*} T f=\int_{S} f(s) \mu(d s) x^{*}, f \in C(S), x^{*} \in \mathfrak{X}^{*}$;

(d) $|T|=\|\mu\|(S)$, the semi-variation of $\mu$ over $S$.

Conversely, if $\mu$ is a set function on $\Sigma$ to $\mathfrak{X}^{* *}$ satisfying ( $a$ ) and (b), then equation $(c)$ defines an operator $T: C(S) \rightarrow \mathfrak{X}$ with norm given by $(d)$, and such that $T^{*} x^{*}=\mu(\cdot) x^{*}$.

Proof. For $E$ in $\Sigma$ let $\mu(E)=T^{* *}\left(\phi_{E}\right)$ where $\phi_{E}$ is that element of $C^{* *}(S)$ defined by the equation $\phi_{E}(\lambda)=\lambda(E)$ for $\lambda$ in $R(S)$. Now the proof proceeds along standard lines and we omit the details.

We now ask when we can be assured that the values of $\mu$ are contained in $\mathfrak{X}$.

3.2. Theorem. If $T$ is a weakly compact operator from $C(S)$ to $\mathfrak{X}$, then there exists a unique vector measure $\mu$ on the Borel sets $\Sigma$ to $\mathfrak{X}$ such that

(a) $T f=\int_{s} f(s) \mu(d s), f \in C(S)$;

(b) $|T|=\|\mu\|(S)$;

(c) $T^{*} x^{*}=x^{*} \mu, \quad x^{*} \in \mathfrak{X}^{*}$.

Conversely, if $\mu$ is a vector measure defined on the Baire sets $\Sigma_{\theta}$ to $\mathfrak{X}$ and if $T$ is defined by $(a)$, then $T$ is a weakly compact operator from $C(S)$ to $\mathfrak{X}$ with norm given by $(b)$ and adjoint operator given by $(c)$.

Proof. If $T$ is weakly compact, then $T^{* *}$ maps $C^{* *}(S)$ into $\mathfrak{X}$ and thus, from the definition of $\mu$, it is clear that $\mu(E)$ is in $\mathfrak{X}$ for every $E$ in $\Sigma$. It follows that $x^{*} \mu$ is in $R(S)$ and $\mu$ is therefore a vector measure. Thus the integral $\int_{S} f(s) \mu(d s)$ exists for $f$ in $C(S)$. From Theorem 3.1 it follows that $x^{*} T=T^{*} x^{*}$. $=x^{*} \mu$. Thus equation (a) follows from Theorem 2.6(f). Conversely, if $\mu: \Sigma_{0} \rightarrow \mathfrak{X}$ 
is a vector measure and if $T$ is defined by (a), then $T^{*}$ maps $x^{*}$ into $x^{*} \mu$. By Lemma $2.3, T^{*}$ is weakly compact. Consequently, $T$ is weakly compact.

The next three results were proved by Grothendieck (7) who used other methods. ${ }^{3}$

3.3. Corollary. If $T$ is a weakly compact operator from $C(S)$ to $\mathfrak{X}$, then $T$ maps weakly fundamental sequences into strongly convergent sequences. Consequently, $T$ maps conditionally weakly compact subsets of $C(S)$ into conditionally strongly compact subsets of $\mathfrak{X}$.

Proof. If $\left\{f_{n}\right\}$ is a weakly fundamental sequence in $C(S)$, then $\left|f_{n}(s)\right|<M$ for some number $M$, and $f_{0}(s)=\lim f_{n}(s)$ exists for each $s \in S$, although $f_{0}$ may not be in $C(S)$. From Theorem 2.8 we conclude that

$$
T f_{n}=\int_{S} f_{n}(s) \mu(d s) \rightarrow \int_{S} f_{0}(s) \mu(d s)
$$

in the norm of $\mathfrak{X}$. This proves the first assertion; the second follows directly.

3.4. Corollary. If $U: \mathfrak{Y} \rightarrow C(S)$ and $T: C(S) \rightarrow \mathfrak{X}$ are weakly compact operators, then $T U: \mathfrak{Y} \rightarrow \mathfrak{X}$ is strongly compact.

3.5. Theorem. ${ }^{4}$ If $T$ is an arbitrary operator on $C(S)$ to $\mathfrak{X}$, and if $\mathfrak{X}$ is weakly complete,,$^{5}$ then there exists a vector measure $\mu_{0}$ defined on the Baire sets $\Sigma_{0}$ of $S$ with values in $\mathfrak{X}$ such that

$$
T f=\int_{S} f(s) \mu_{0}(d s), \quad f \in C(S) .
$$

Consequently, an arbitrary operator from $C(S)$ to a weakly complete Banach space is weakly compact.

Proof. Let $\mu: \Sigma \rightarrow \mathfrak{X}^{* *}$ be the set function whose existence was established in Theorem 3.1. We shall show that $\mu$ maps $\Sigma_{0}$ into $\mathfrak{X}$. Let $f$ be a bounded function in the first Baire class; the reader may readily verify that there exists a bounded sequence $\left\{f_{n}\right\}$ in $C(S)$ which converges pointwise to $f$. Then $\left\{f_{n}\right\}$ and $\left\{T f_{n}\right\}$ are weakly fundamental; hence $\left\{T f_{n}\right\}$ converges weakly to an element of $\mathfrak{X}$. If $f$ is regarded as an element of $C^{* *}(S)$, it is evident that $\left\{T f_{n}\right\}$ converges to $T^{* *} f$ in the $\mathfrak{X}^{*}$ topology of $\mathfrak{X}^{* *}$. We conclude that $T^{* *} f \in \mathfrak{X}$. By induction, $T^{* *}$ maps the bounded Baire functions into $\mathfrak{X}$; in particular, this is true for the characteristic functions of Baire sets. Hence $\mu$ maps $\Sigma_{0}$ into $\mathfrak{X}$. Since the Baire

${ }^{3}$ Although Grothendieck (7) did not employ the representation for weakly compact operators given above, he noted the one-to-one correspondence between weakly compact operators on $C(S)$ to $\mathfrak{X}$ and vector measures on the Baire subsets of $S$ to $\mathfrak{X}$. Theorem 3.2 can be proved using results in (7) but there is some interest in the measure theoretic approach given here.

${ }^{4}$ Gelfand (6) showed that an operator on $C[0,1]$ to a weakly complete space can be represented as an integral with respect to a vector function of bounded variation. The final conclusion of the present theorem was announced by Pettis (12); it was also proved by Grothendieck.

${ }^{5} \mathrm{~A}$ Banach space $\mathfrak{X}$ is weakly complete if every weakly fundamental sequence in $\mathfrak{X}$ converges weakly to an element of $\mathfrak{X}$. 
sets are sufficient to integrate continuous functions, we have the representation

$$
T f=\int_{S} f(s) \mu_{0}(d s), \quad f \in C(S),
$$

where $\mu_{0}$ is the restriction of $\mu$ to $\Sigma_{0}$. The second assertion follows from the converse part of Theorem 3.2.

3.6. Corollary. An arbitrary continuous linear mapping from an abstract $M$-space to an abstract $L$-space is weakly compact.

Proof. The terminology is that used by Kakutani $(\mathbf{9} ; \mathbf{1 0})$. We observe that the conjugate of an abstract $L$-space is an abstract $M$-space with a unit element and hence is isometrically isomorphic to $C(S)$ for some compact Hausdorff space $S$ (10, pp. 1023, 998). Further, the adjoint of an $M$-space is an $L$-space and is therefore weakly complete $(\mathbf{1 0}$, p. $1021 ; \mathbf{9}$, p. 537$)$. Thus $T^{*}$ and $T$ are weakly compact.

3.7. Corollary. ${ }^{6}$ If $T$ is a weakly compact map of an abstract $M$-space (or an abstract L-space) into itself, then $T^{2}$ is strongly compact.

Proof. The second conjugate of an abstract $M$-space is an abstract $M$-space with unit (10, p. 1023) and hence is isometrically isomorphic to $C(S)$. From Corollary 3.4 we conclude that $\left(T^{* *}\right)^{2}$ and hence $T^{2}$ is strongly compact. The second assertion follows from the first and the fact that the conjugate of an $L$-space is an $M$-space.

We now turn to the representation of a compact operator on $C(S)$.

3.8. Theorem. An operator $T: C(S) \rightarrow \mathfrak{X}$ is compact if and only if the vector measure $\mu: \Sigma \rightarrow \mathfrak{X}$ corresponding to it (as in Theorem 3.2 ) takes its values in a compact subset of $\mathfrak{X}$.

Proof. If $T^{* *}$ is compact, then by the construction of $\mu$, we see that the condition is necessary. To see that it is sufficient, it is enough to prove that the set $\Omega$ of all sums of the form

$$
\sum_{i=1}^{n} \alpha_{i} \mu\left(E_{i}\right)
$$

where the $\left\{E_{i}\right\}$ are disjoint and $\left|\alpha_{i}\right| \leqslant 1$, is a totally bounded set in $\mathfrak{X}$. Let $\epsilon>0$ be given and let $M$ be the semi-variation of $\mu$ on $S$. Select a set $\left\{\beta_{1}, \ldots, \beta_{p}\right\}$ of complex numbers with $\left|\beta_{i}\right| \leqslant 1$ such that if $|\alpha| \leqslant 1$ then there exists a $\beta_{i}=\beta(\alpha)$ with $|\beta(\alpha)-\alpha|<\epsilon / 2 M$. Let $\left\{F_{1}, \ldots, F_{q}\right\} \subset \Sigma$ be such that if

${ }^{6}$ This result was proved for a concrete $L$-space by Dunford and Pettis (3), and also by Phillips (13), by explicitly representing the weakly compact operators. It implies that if an abstract $M$ - or $L$-space contains an infinite dimensional reflexive subspace, then there is no bounded projection mapping onto this subspace. 
$E \in \Sigma$, then there is an $F_{k}=F(E)$ with $|\mu(F(E))-\mu(E)|<\epsilon / 2 p$. Then from the definition of the semi-variation

$$
\left|\sum_{i=1}^{n} \alpha_{i} \mu\left(E_{i}\right)-\sum_{i=1}^{n} \beta\left(\alpha_{i}\right) \mu\left(E_{i}\right)\right|=\left|\sum_{i=1}^{n}\left\{\alpha_{i}-\beta\left(\alpha_{i}\right)\right\} \mu\left(E_{i}\right)\right| \leqslant \frac{\epsilon}{2 M} \cdot M=\frac{1}{2} \epsilon .
$$

Further,

$$
\sum_{i=1}^{n} \beta\left(a_{i}\right) \mu\left(E_{i}\right)
$$

can be written as a sum

$$
\sum_{j=1}^{p} \beta_{j} \mu\left(E_{j}^{\prime}\right),
$$

with $\left\{E_{j}^{\prime}\right\}$ a disjoint family in $\Sigma$. Thus

$$
\left|\sum_{j=1}^{p} \beta_{j} \mu\left(E_{j}{ }^{\prime}\right)-\sum_{j=1}^{p} \beta_{j} \mu\left(F\left(E_{j}{ }^{\prime}\right)\right)\right| \leqslant \sum_{j=1}^{p}\left|\mu\left(E_{j}{ }^{\prime}\right)-\mu\left(F\left(E_{j}{ }^{\prime}\right)\right)\right|<p \cdot \frac{\epsilon}{2 p}=\frac{1}{2} \epsilon \text {. }
$$

We have shown that each element in $\Re$ can be approximated within $\epsilon$ by sums of the form

$$
\sum_{j=1}^{p} \beta_{j} \mu\left(F_{k_{j}}\right)
$$

so that $\Omega$ is totally bounded.

4. Special cases. It is easy (2) to give representations of operators which map a Banach space into $C(Q)$, where $Q=\{t\}$ is a compact Hausdorff space. Thus an operator $T: C(S) \rightarrow C(Q)$ may be studied from two standpoints. We now give kernel representations of operators in these spaces. We remark that in the case that $S=Q=[a, b]$, the general and compact operator was represented by Radon (14), and a representation of the weakly compact operator similar to Theorem 4.2 was given by Sirvint (16). In what follows $S$ and $Q$ are compact Hausdorff spaces.

4.1 ThEOREM. If $T$ is an arbitrary bounded operator from $C(S)$ to $C(Q)$ then $T$ can be represented by the formula

$$
(T f)(t)=\int_{S} f(s) K(d s, t), \quad f \in C(S), \quad t \in Q ;
$$

where $K$ is defined on $\Sigma \times Q$ to the scalar field and satisfies

(i) $K(., t) \in R(S)$ for each $t \in Q$;

(ii) the integral in $(*)$ is in $C(Q)$ for each $f \in C(S)$;

(iii) $\sup _{t \in Q}|K(\cdot, t)|=|T|<\infty$.

Conversely, if $K$ satisfies these conditions, the operator defined by $\left(^{*}\right)$ maps $C(S)$ into $C(Q)$ and has norm given by (iii). 
4.2. THEOREM. If $T$ is a weakly compact operator from $C(S)$ to $C(Q)$ then $T$ can be represented by formula $\left({ }^{*}\right)$, where $K$ satisfies (i) and

(ii') $K(E,.) \in C(Q)$ for each $E \in \Sigma$;

(iii') $|K(E, t)| \leqslant|T|<\infty$ for $E \in \Sigma, t \in Q$.

Conversely if $K$ satisfies these conditions, the operator defined by $\left(^{*}\right)$ is a weakly compact map of $C(S)$ into $C(Q)$ and has norm given by (iii).

Proof. It is easily seen from Theorem 3.2 that a weakly compact operator must have this form. To prove the converse, let $\mu: \Sigma \rightarrow C(Q)$ be defined by $\mu(E)=K(E, \cdot)$. Then if $\left\{E_{i}\right\}$ is a disjoint sequence in $\Sigma$, it follows from (i) that

$$
K\left(\bigcup_{i=1}^{\infty} E_{i}, t\right)=\sum_{i=1}^{\infty} K\left(E_{i}, t\right),
$$

and from the well-known criterion for weak convergence in $C(Q)$ that $x^{*} \mu$ is countably additive for any $x^{*} \in C^{*}(Q)$. With (iii') this implies that $\mu$ is a vector measure in the sense of Definition 2.1.

For some purposes the following representation may be more convenient. It is derived readily from the above and the Radon-Nikodým theorem.

4.3. THEOREM. If $T: C(S) \rightarrow C(Q)$ is weakly compact, then $T$ can be represented by the formula

$$
(+) \quad(T f)(t)=\int_{S} f(s) k(s, t) \nu(d s), \quad f \in C(S), \quad t \in Q \text {; }
$$

where $\nu$ is a positive Borel measure on $(S, \Sigma)$ and $k$ is a function from $S \times Q$ to the scalar field such that

(a) $k(\cdot, t) \in L(S, \Sigma, \nu)$ for each $t \in Q$;

(b) $\int_{E} k(s, \cdot) \nu(d s) \in C(Q)$ for each $E \in \Sigma$;

(c) $\sup _{t \in Q} \int_{S}|k(s, t)| \nu(d s)=|T|<\infty$.

Conversely, if $k$ and $\nu$ satisfy these conditions then $(+)$ defines a weakly compact operator.

4.4. Theorem. If $T: C(S) \rightarrow C(Q)$ is compact, then $T$ can be represented by the formula $(+)$ where $\nu$ is a positive Borel measure on $(S, \Sigma)$ and $k$ satisfies (a), (c) and

$$
\lim _{t \rightarrow t_{0}} \int_{S}\left|k(s, t)-k\left(s, t_{0}\right)\right| \nu(d s)=0,
$$

Conversely, if $k$ and $\nu$ satisfy these conditions then $(+)$ defines a compact operator. 


\section{REFERENCES}

1. A. Alexiewicz, On sequences of operations (I), Studia Math., 11 (1949), 1-30.

2. R. G. Bartle, On compactness in functional analysis, Trans. Amer. Math. Soc., 79 (1955).

3. N. Dunford and B. J. Pettis, Linear transformations on summable functions, Trans. Amer. Math. Soc., 47 (1940), 323-392.

4. N. Dunford and J. Schwartz, Spectral theory; forthcoming book.

5. W. F. Eberlein, Weak compactness in Banach spaces (I), Proc. Nat. Acad. Sci. U.S.A., 33 (1947), 51-53.

6. I. Gelfand, Abstrakte Funktionen und lineare Operatoren, Mat. Sbornik (4) 46 (1938), 235-284.

7. A. Grothendieck, Sur les applications linéares faiblement compactes d'espace du type $C(K)$, Can. J. Math., 5 (1953), 129-173.

8. P. R. Halmos, Measure theory (New York, 1950).

8a. E. Hille, Functional analysis and semi-groups (Amer. Math. Soc. Colloquium Publications, vol. 31, 1948).

9. S. Kakutani, Concrete representation of abstract $(L)$-spaces and the mean ergodic theorem, Ann. Math. (2) 42 (1941), 523-537.

10. - Concrete representation of abstract (M)-spaces, Ann. Math. (2) 42 (1941), 994-1024.

11. B. J. Pettis, On integration in vector spaces, Trans. Amer. Math. Soc., 44 (1938), 277-304.

12. - - Absolutely continuous functions in vector spaces (Abstract), Bull. Amer. Math. Soc., 45 (1939), 677.

13. R. S. Phillips, On linear transformations, Trans. Amer. Math. Soc., 48 (1940), 516-541.

14. J. Radon, Über lineare Funktionaltransformationen und Funktionalgleichungen, Sitzber. Akad. Wiss. Wien, 128 (1919), 1083-1121.

15. S. Saks, On some functionals, Trans. Amer. Math. Soc., 85 (1933), 549-556, 965-970.

16. G. Sirvint, Weak compactness in Banach spaces, Studia Math., 11 (1949), 71-94.

\section{Yale University}

